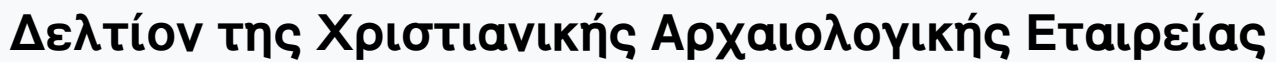

Tó 28 (2007)

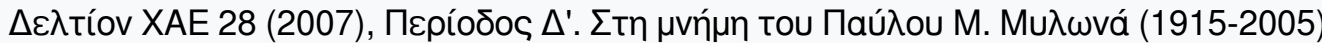

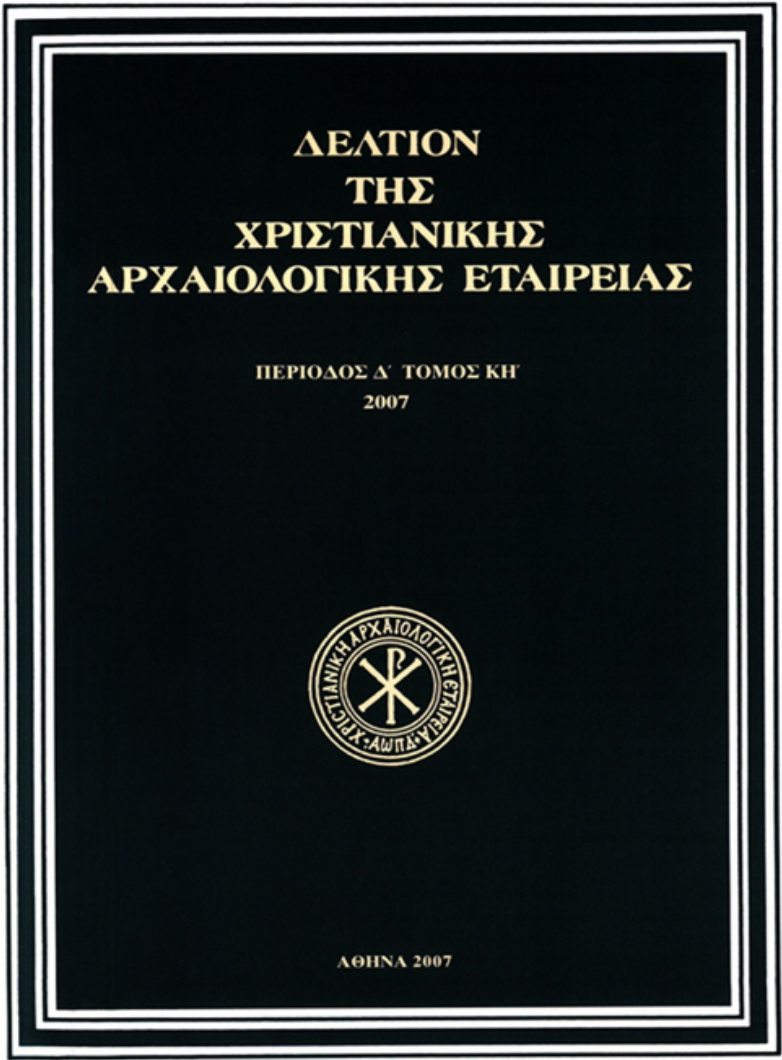

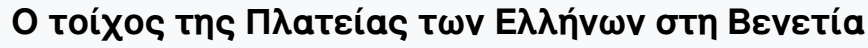

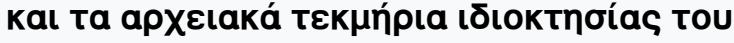

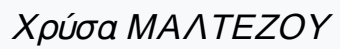

doi: $10.12681 /$ dchae.582

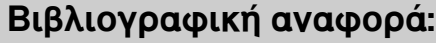

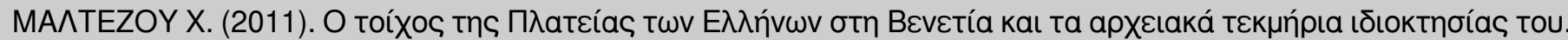

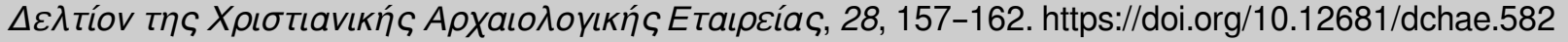




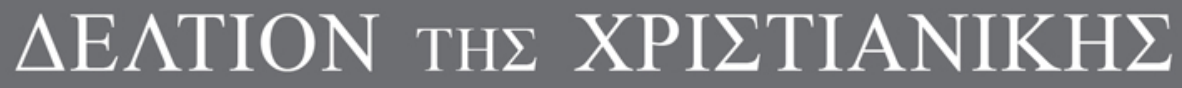 APXAIO $\Lambda$ OГIKH $\Sigma$ ETAIPEIA $\Sigma$}

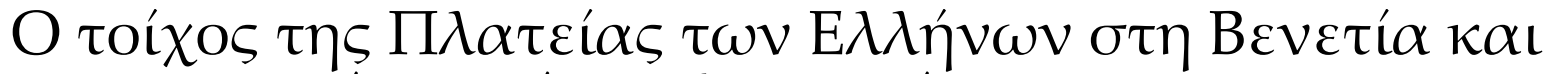

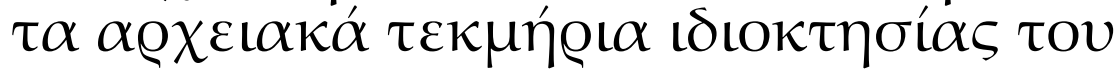

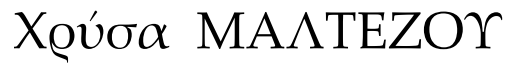

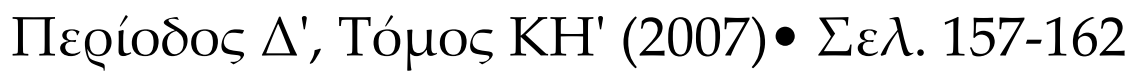
A@HNA 2007 


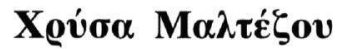

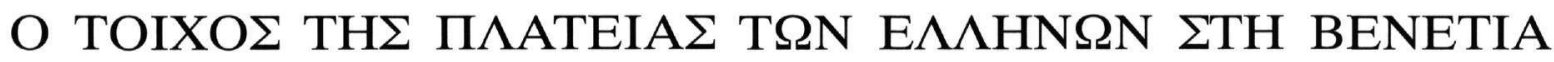 KAI TA APXEIAKA TEKMHPIA I $\triangle I O K T H \Sigma I A \Sigma$ TOY}

${ }^{\mathrm{c}} \mathrm{H}$

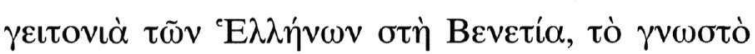

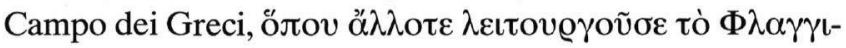

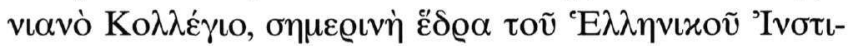

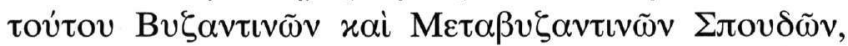

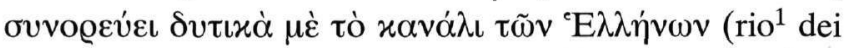

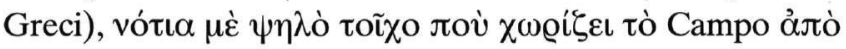

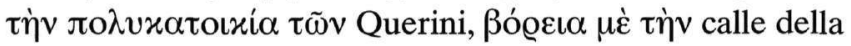

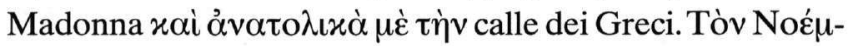

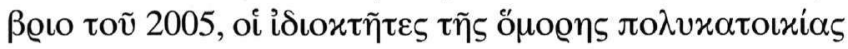

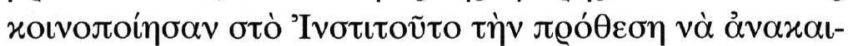

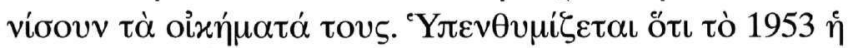

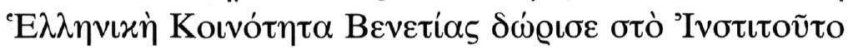

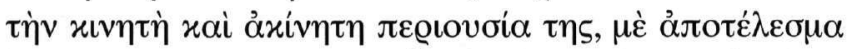

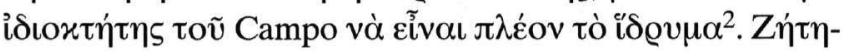

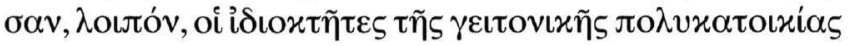

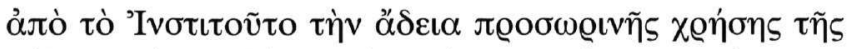

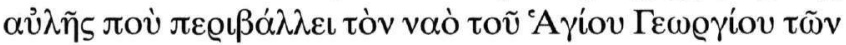

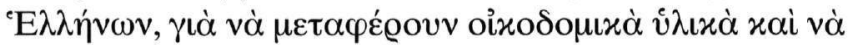

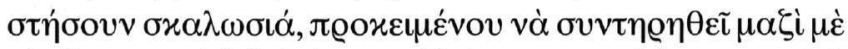

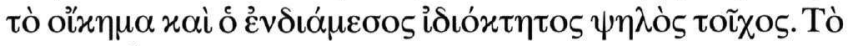

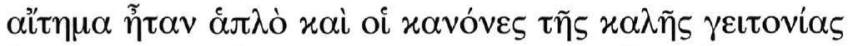

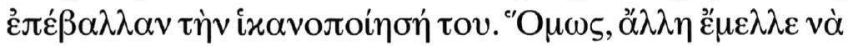

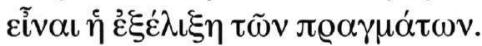

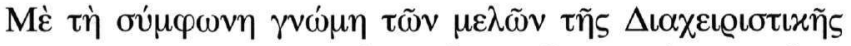

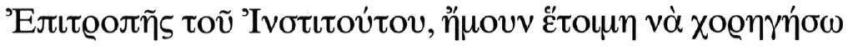

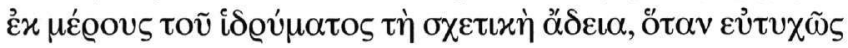

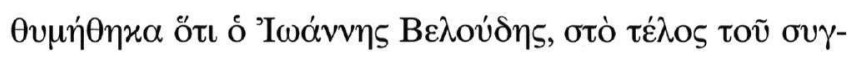

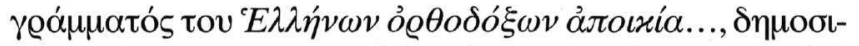

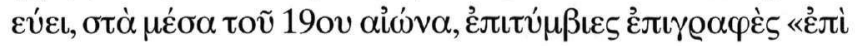

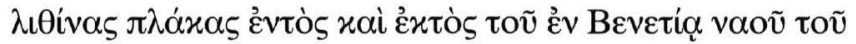

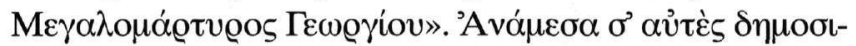

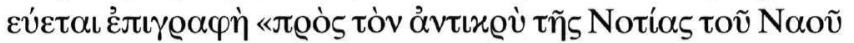

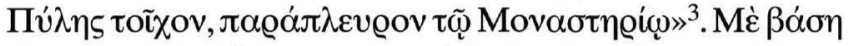

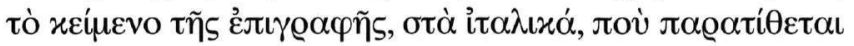

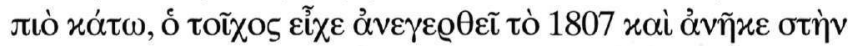

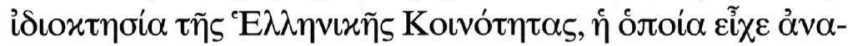

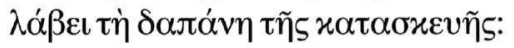

Muro di proprietà assoluta di questa/ Nazione Greca/ ricostruito l' anno 1807/ con li proprj suoi danari/ in virtù dell'acordo formato/ con la famiglia Querini/li 24 M(ar)zo anno su$d($ et)to/ ed aprovato dal Consiglio municipale de' Savj/ con decreto dei 24 Aprile susseguente / in forza del quale si fece apporrel la p(rese)nte lapide a perpetua memoria.

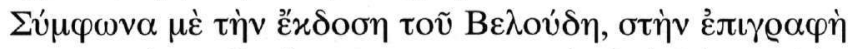

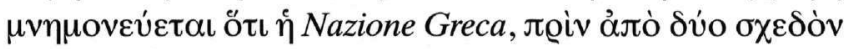

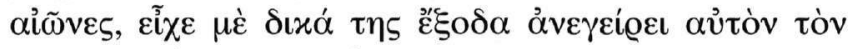

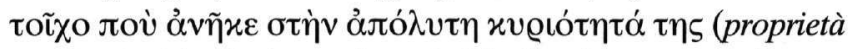

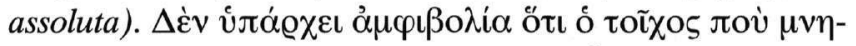

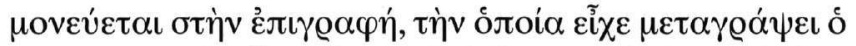

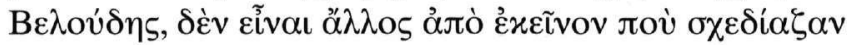

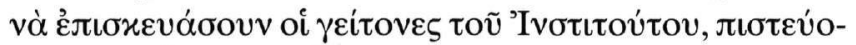

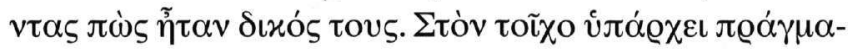

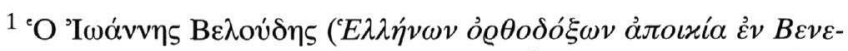

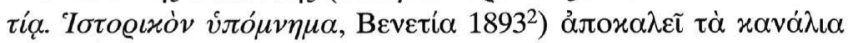

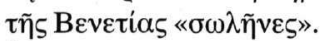

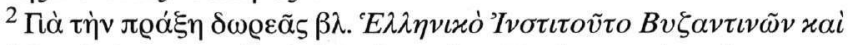

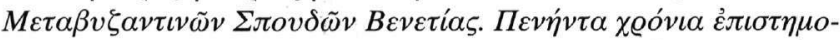

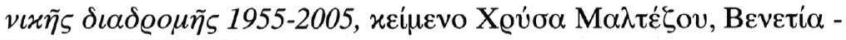
'A $\theta$ ív $2005,11-13$.

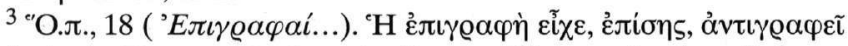

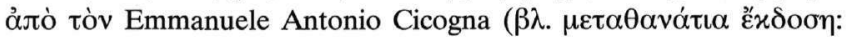
Corpus delle Iscrizioni di Venezia e delle isole della laguna veneta di Emmanuele Antonio Cicogna, opera compilata da Piero Pazzi con il con-
}

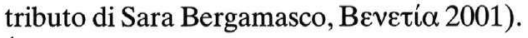

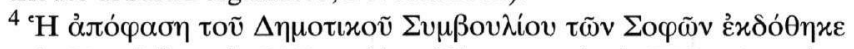

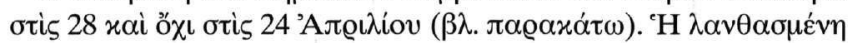

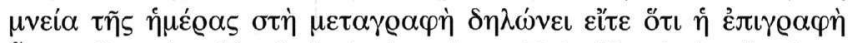

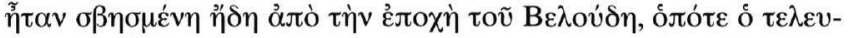

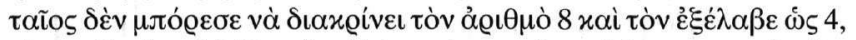

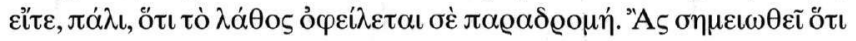

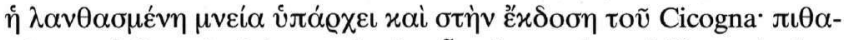

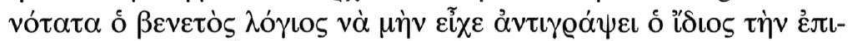

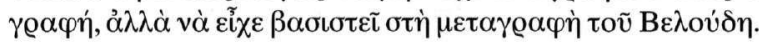




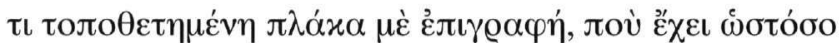

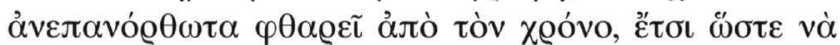

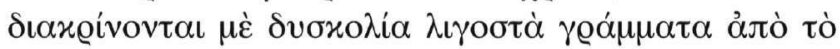

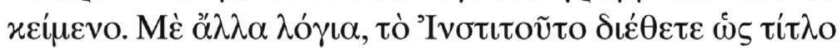

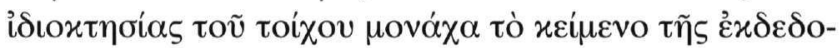

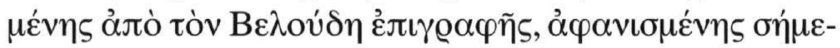

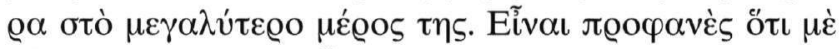

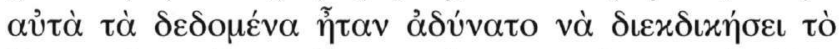

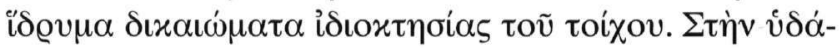

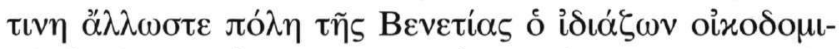

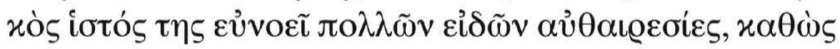

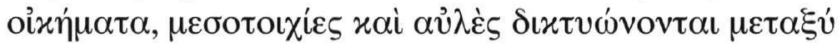

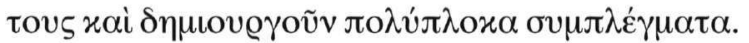

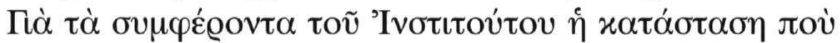

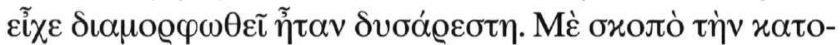

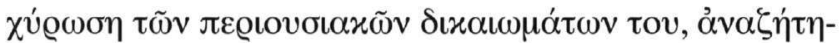

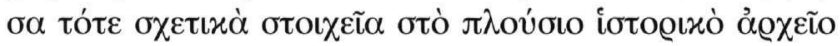

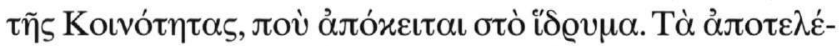

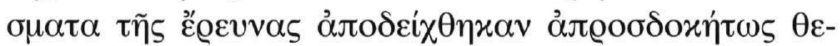

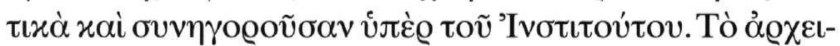

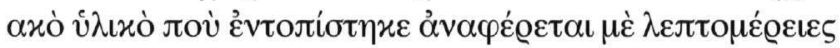

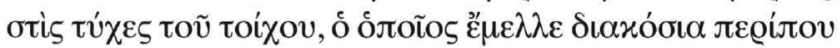

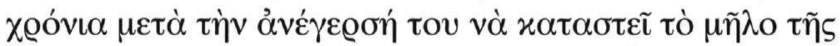

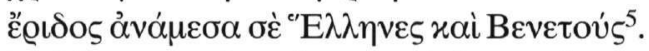

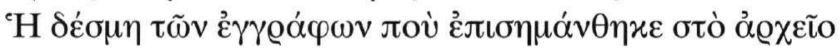

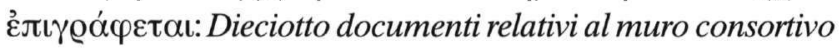
colla casa Querini, erretto a proprie spese dalla Nazione Greca,

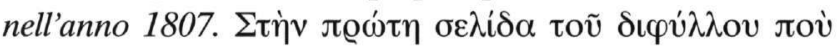

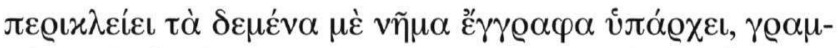
$\mu \varepsilon \dot{v} \eta \mu \varepsilon \dot{~ \tau o ̀ ~ \chi \varepsilon ́ \varrho \iota ~ \tau o v ̃ ~ K \omega v \sigma \tau \alpha v \tau i v o v ~ K \alpha \beta \alpha ́ x o v, ~ \alpha ’ \varrho \chi \varepsilon เ o-~}$

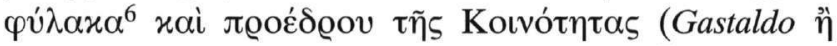

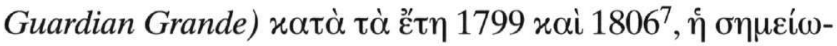
oๆ: Dissegni, supliche, documenti, decreti del Consiglio Municipale de' Savij, ed accordo seguito colla famiglia Querini nell' anno 1807, sotto il guardianato del signor Costantino Cavaco per il muro da stroppo, situato al lato destro della chiesa e

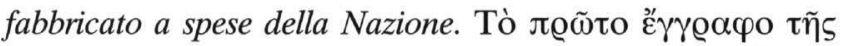

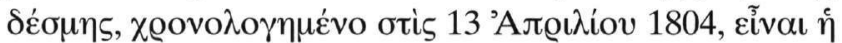

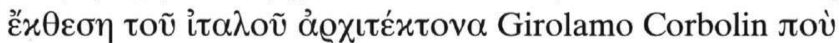

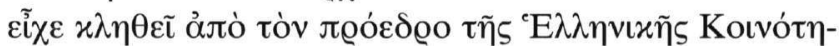

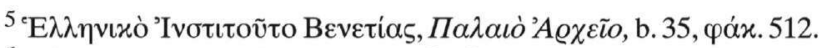

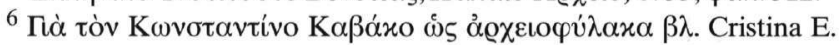
Papacosta - Francesca Cavazzana Romanelli, «La Confraternita dei Greci di Venezia e il suo archivio. Un modello condiviso di organizza-

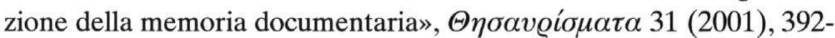

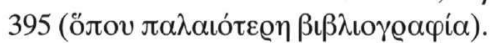

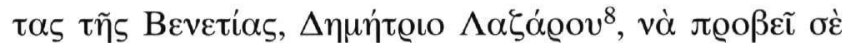

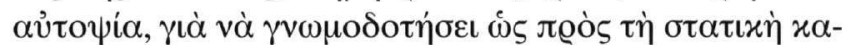

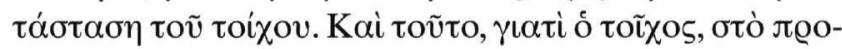

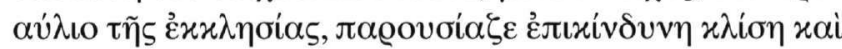

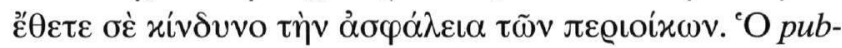

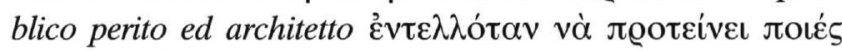

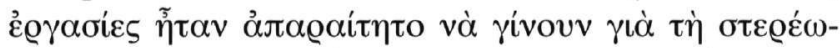

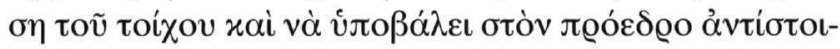

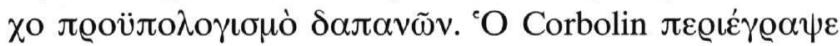

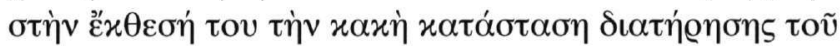

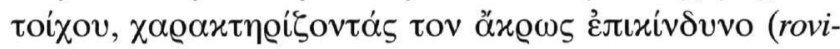

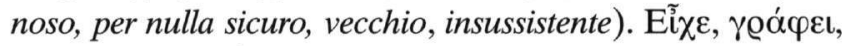

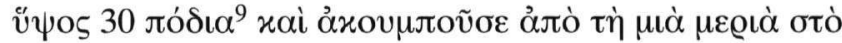

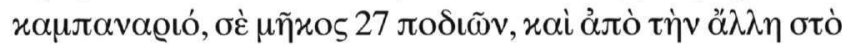

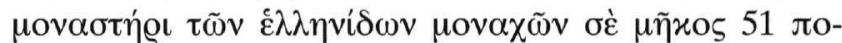

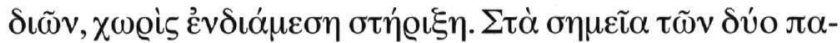

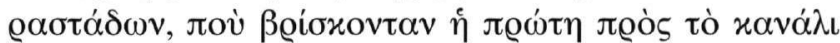

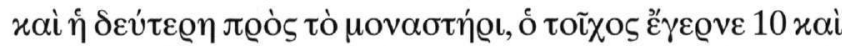

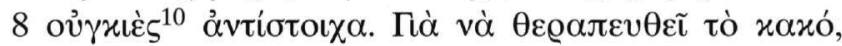

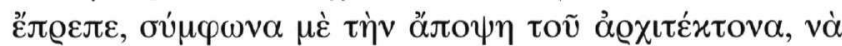

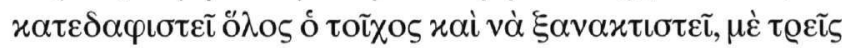

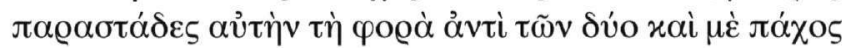

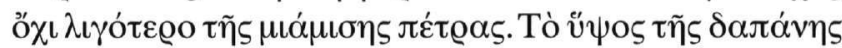

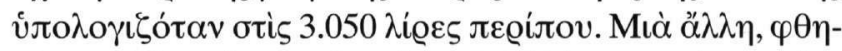

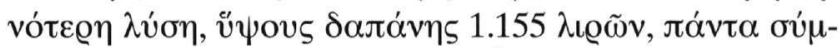

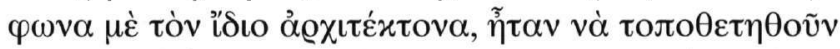

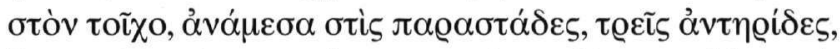

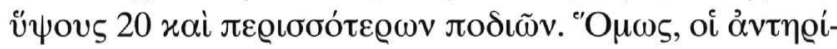

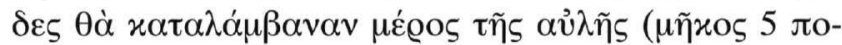

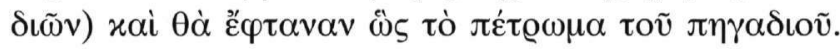

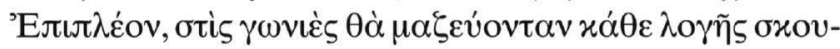

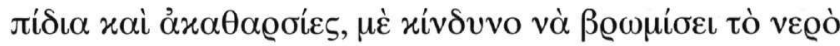

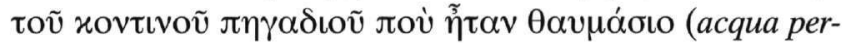

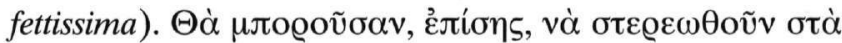

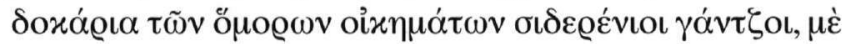

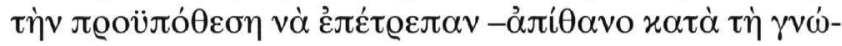

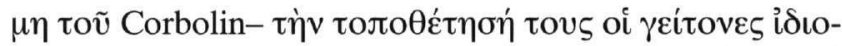

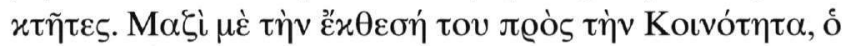

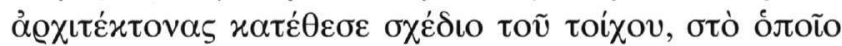

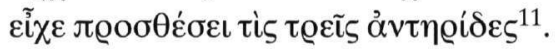

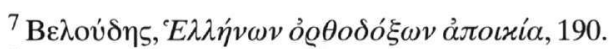

$8{ }^{\circ} \mathrm{O} . \pi$.

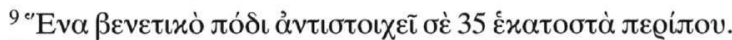

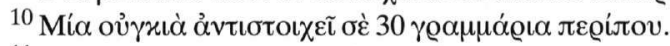

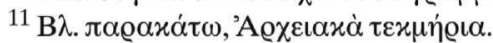




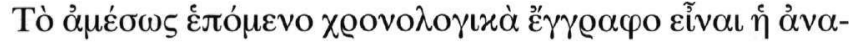

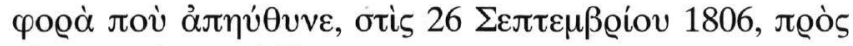

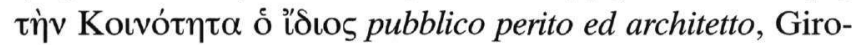

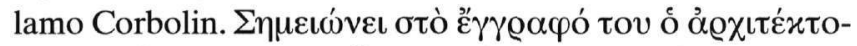

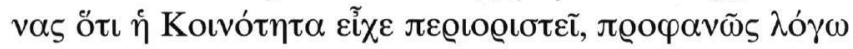

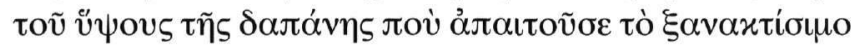

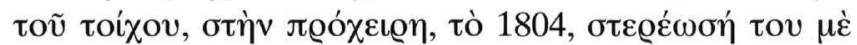

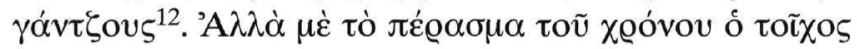

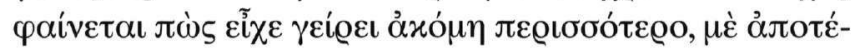

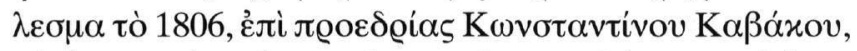

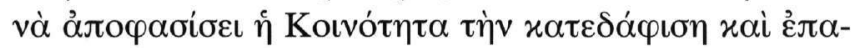

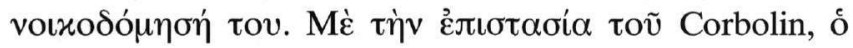

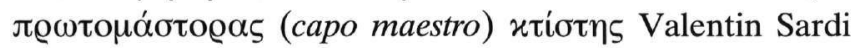

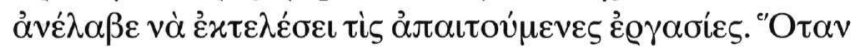

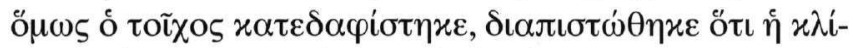

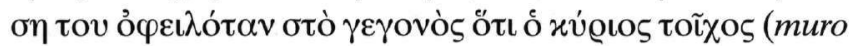

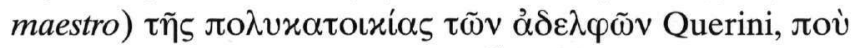

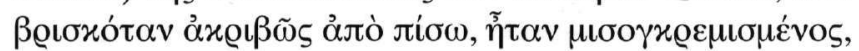

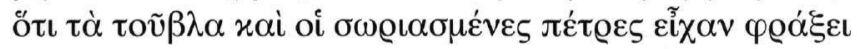

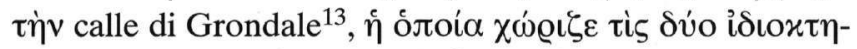

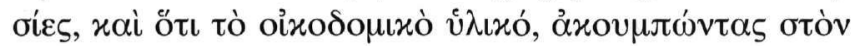

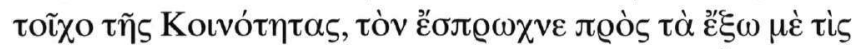

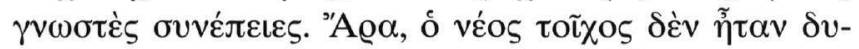

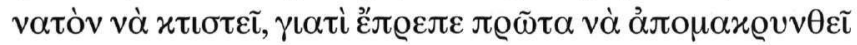

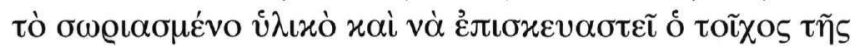

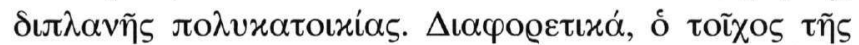

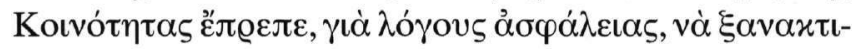

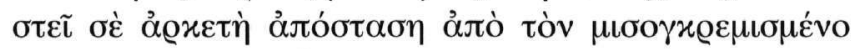

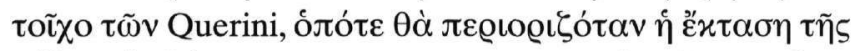

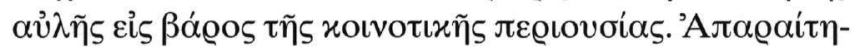

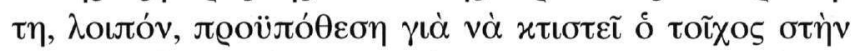

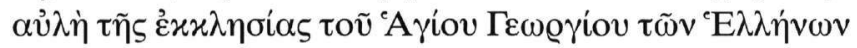

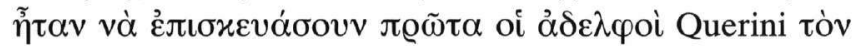

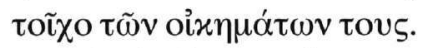

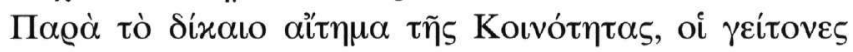

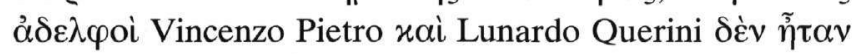

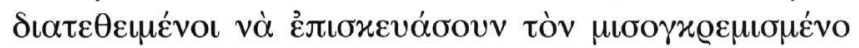

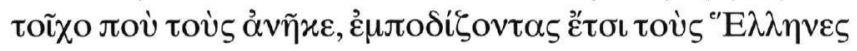

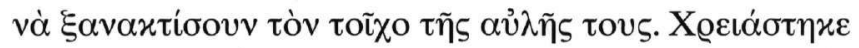

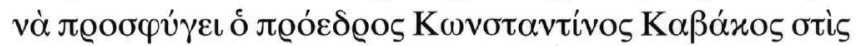

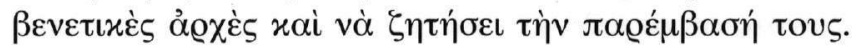

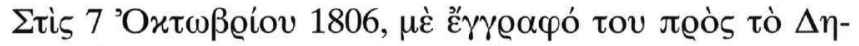

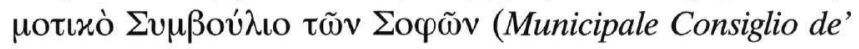

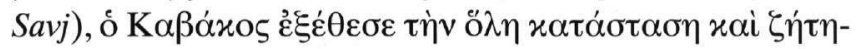

12 ... nel maggio 1804 è stato provvisoriamente assicurato con varie punte assicurate da giazie ed altre punte in piedi, come in simili circostanze si usa

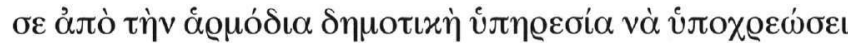

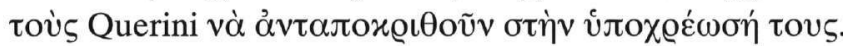

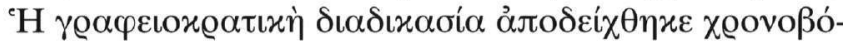

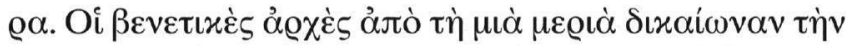

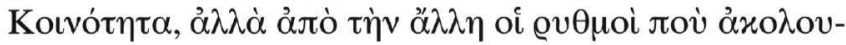

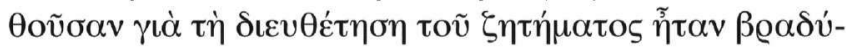

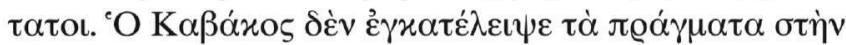

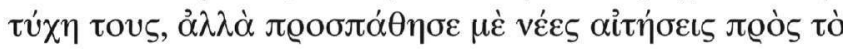

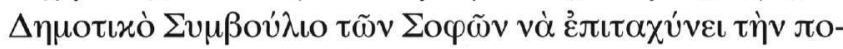

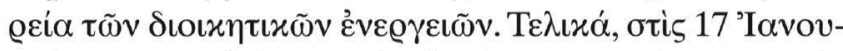

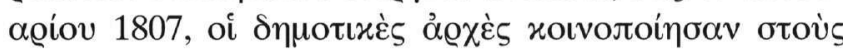

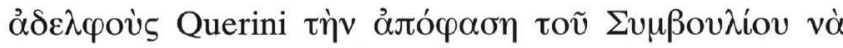

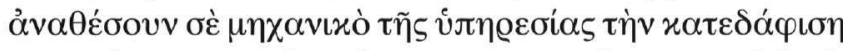

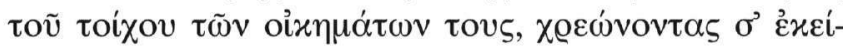

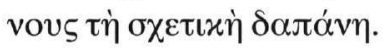

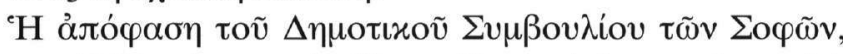

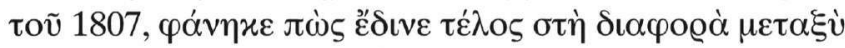

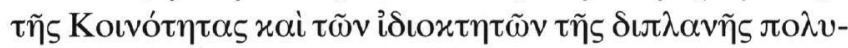

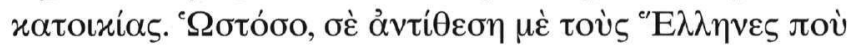

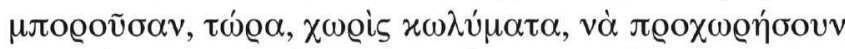

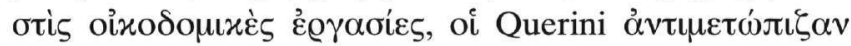

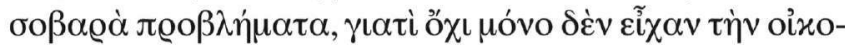

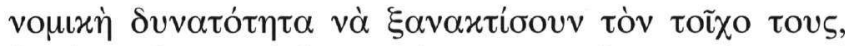

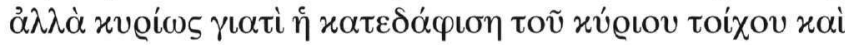

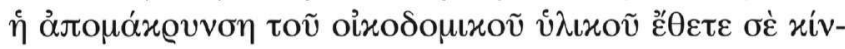

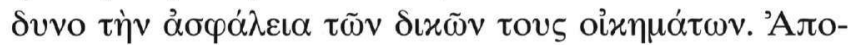

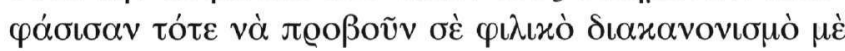

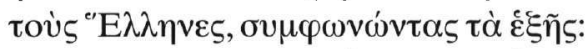

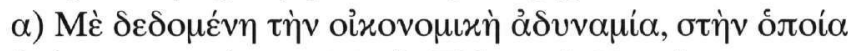

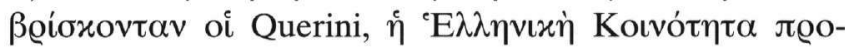

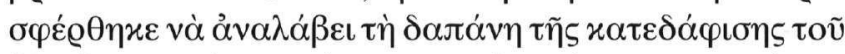

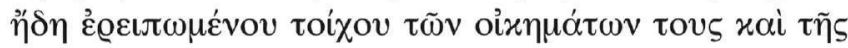

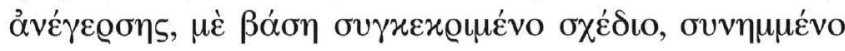

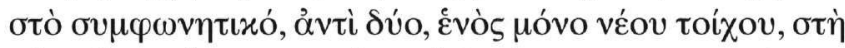

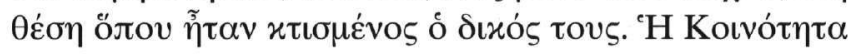

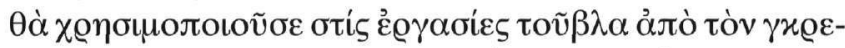

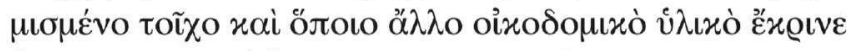

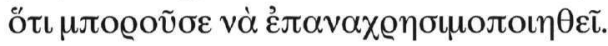

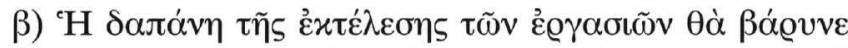

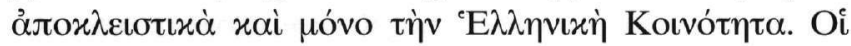

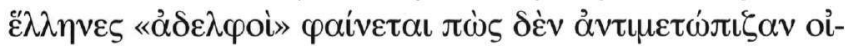

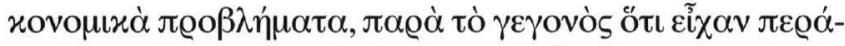

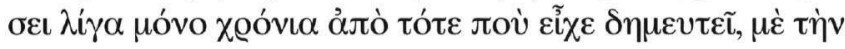

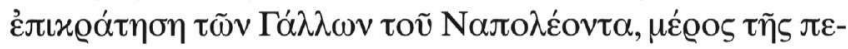

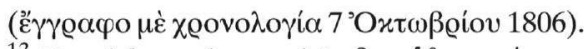

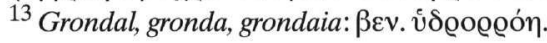




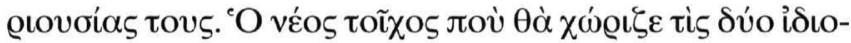

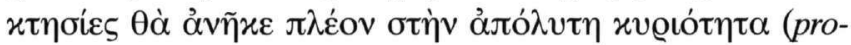

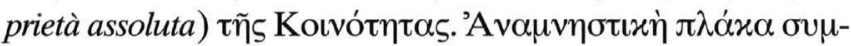

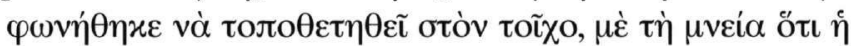

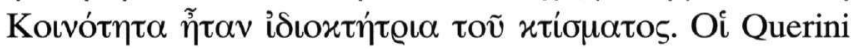

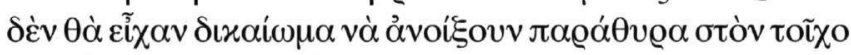

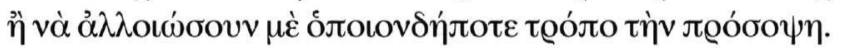

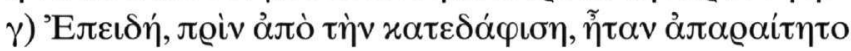

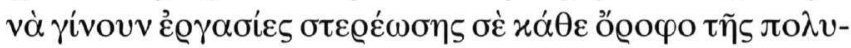

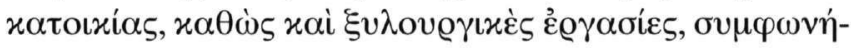

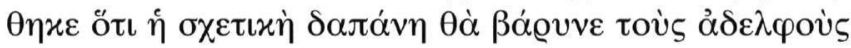
Querini.

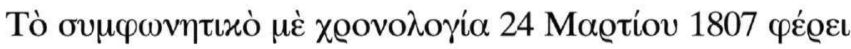

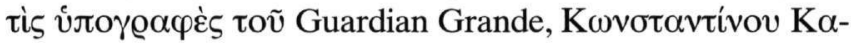

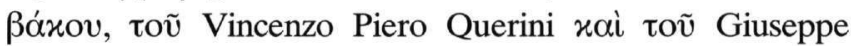

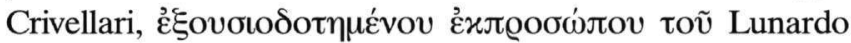

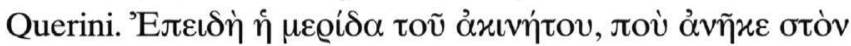

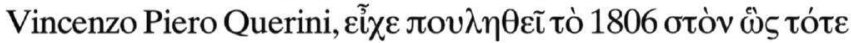

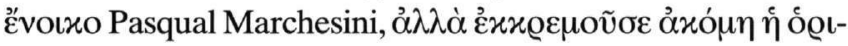

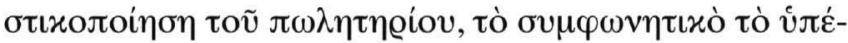

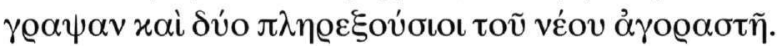

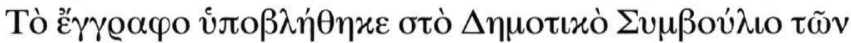

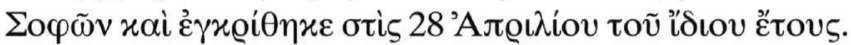

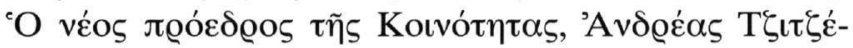

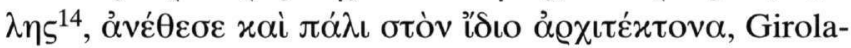

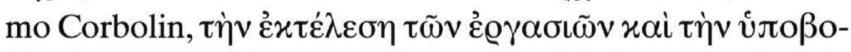

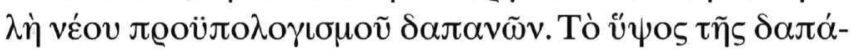

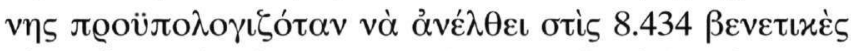

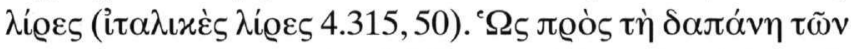

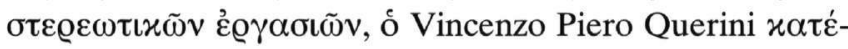

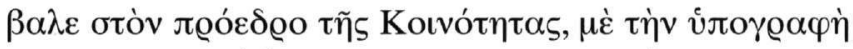

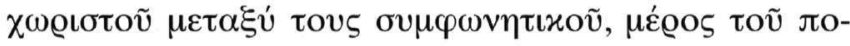

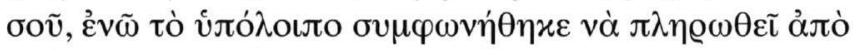

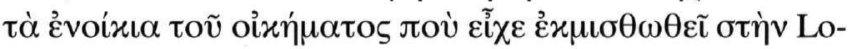
dovica Sirocovich.

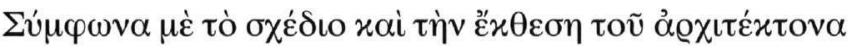

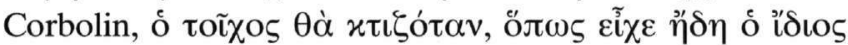

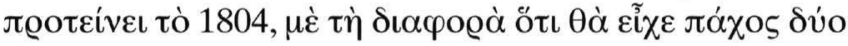

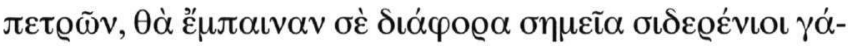

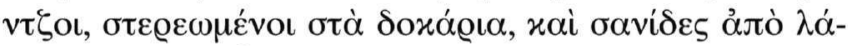

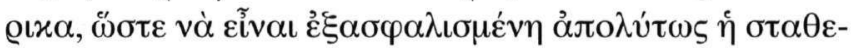

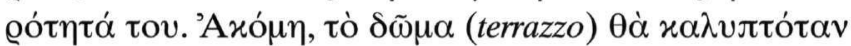

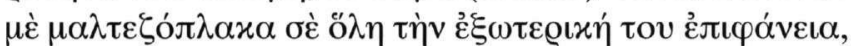

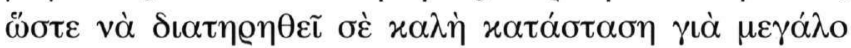

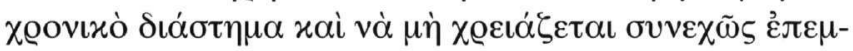

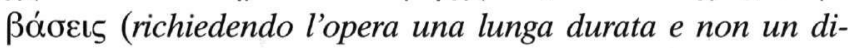

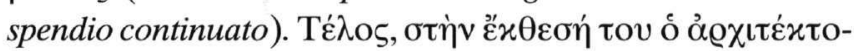

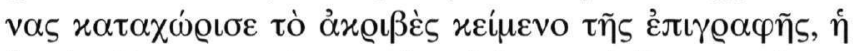

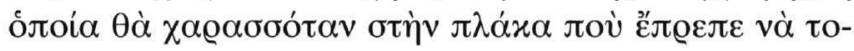

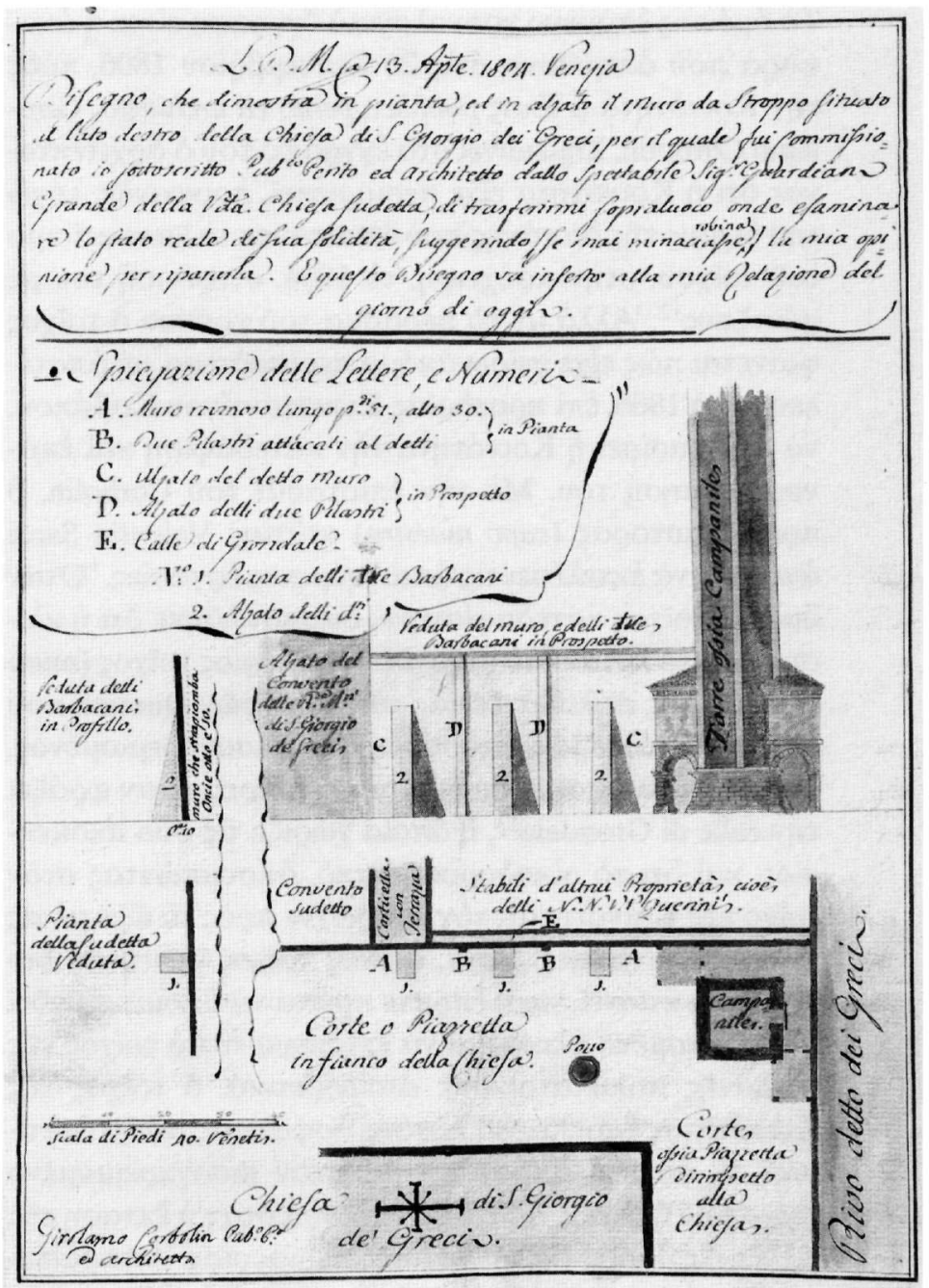

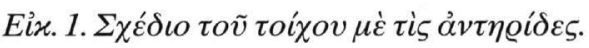

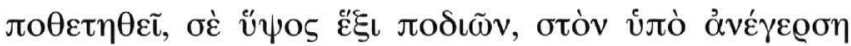

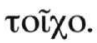

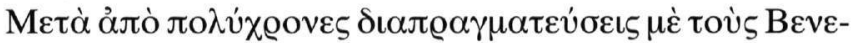

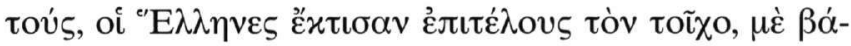

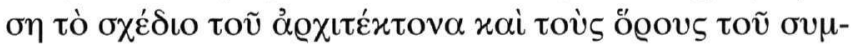

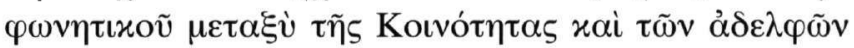

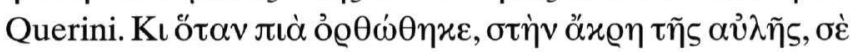

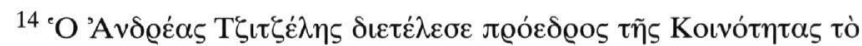

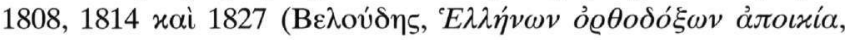
190). 


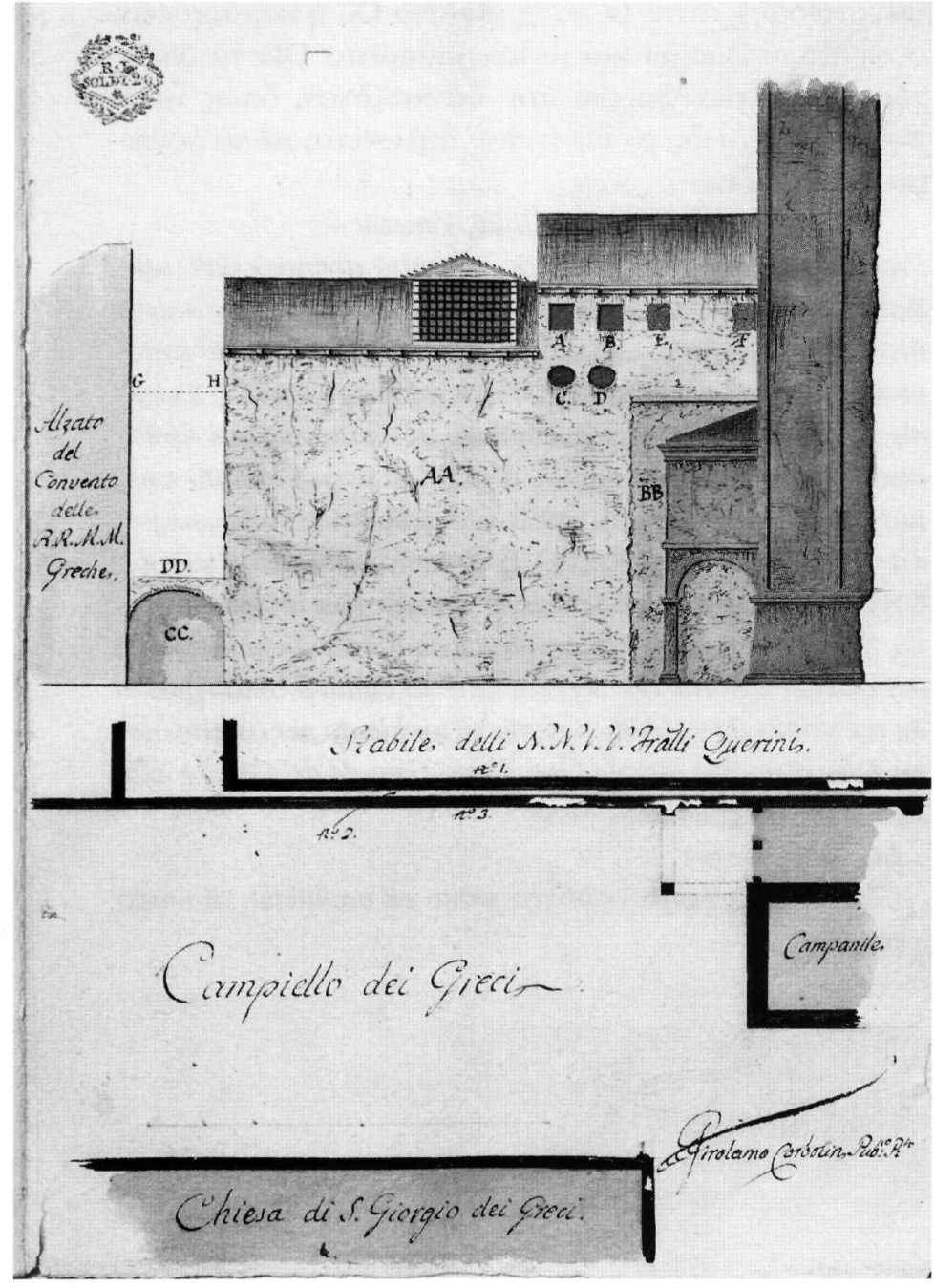

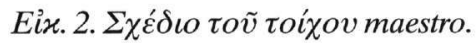

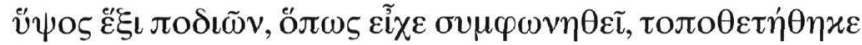

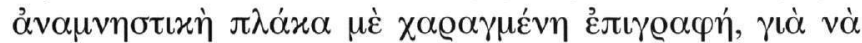
$\delta \eta \lambda \omega ́ v \varepsilon l:$ la proprietà ed assoluto diritto del muro stesso di ragione della Nazione, per l'effetto che in qualunque tempo avvenire per qualunque si sia pretesto, anche se lo stabile Querini venisse demolito, non possa essere turbata la di Lei assoluta proprietà tanto del muro medesimo che dell'aria relativa ... Oi

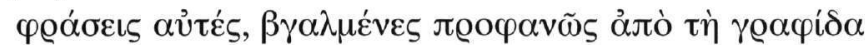

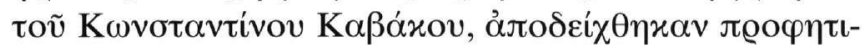

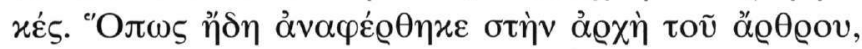

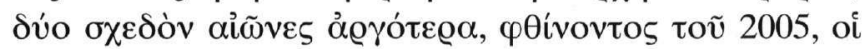

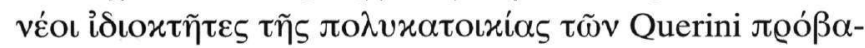

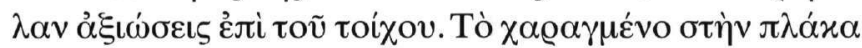

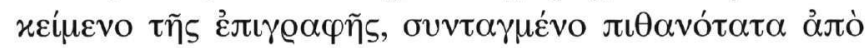

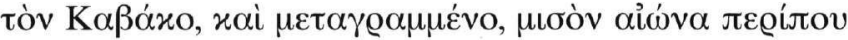

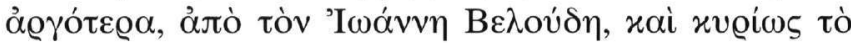

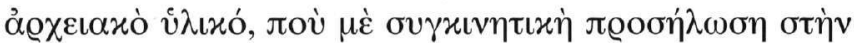

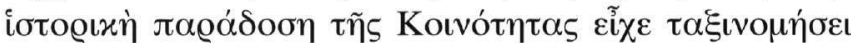

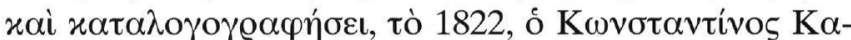

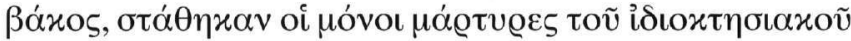

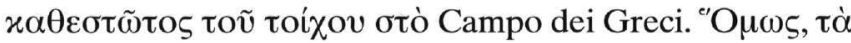

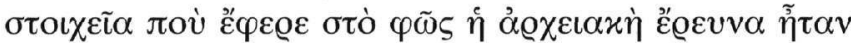

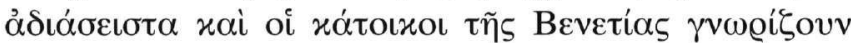

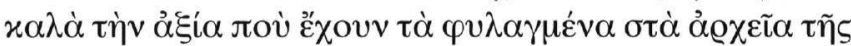

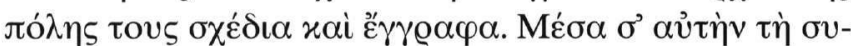

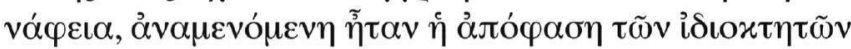

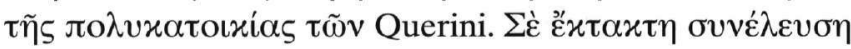

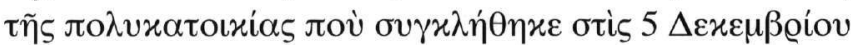

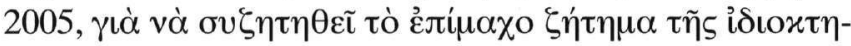

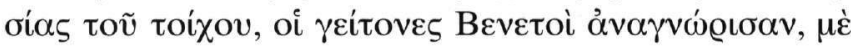

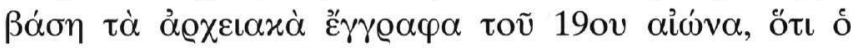

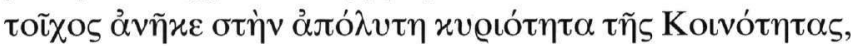

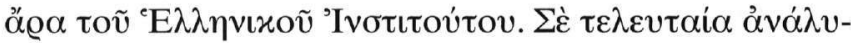

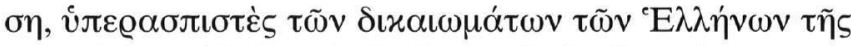

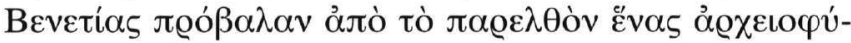

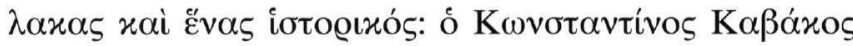

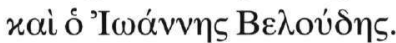

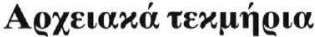

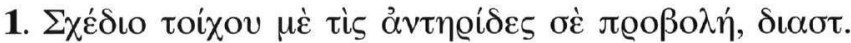

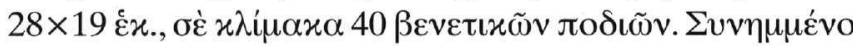

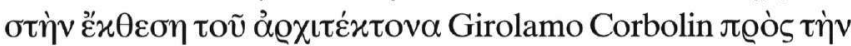

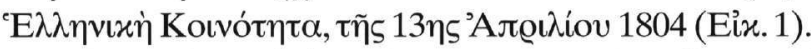

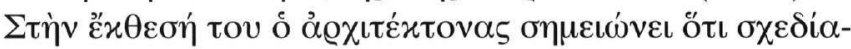

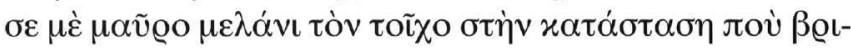

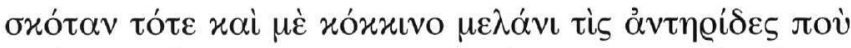

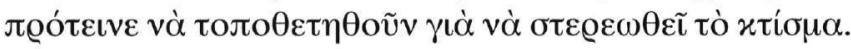

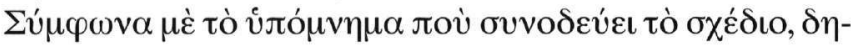

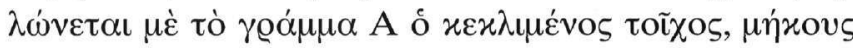

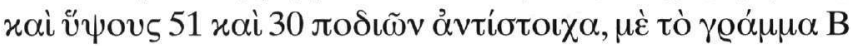

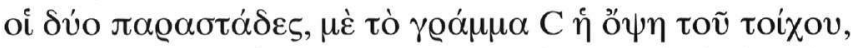

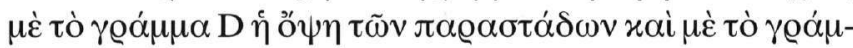

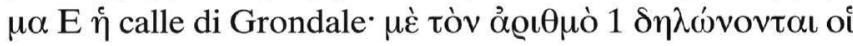

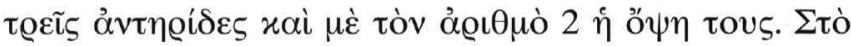

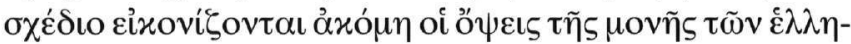

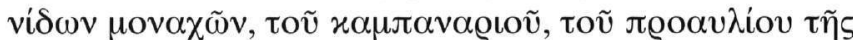

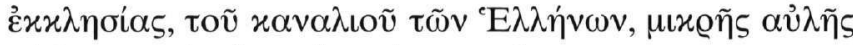

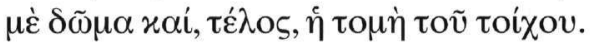

Laus Deo Maximo a 13 aprile 1804, Venezia

Disegno che dimostra in pianta ed in alzato il muro da stroppo, situato al lato destro della chiesa di San Giorgio dei Greci, per il quale fui commissionato io sottoscritto, pubblico perito ed 
architetto, dallo spettabile signor Guardian Grande della veneranda chiesa sudetta di trasferirmi sopraluoco, onde esaminare lo stato reale di sua solidità, suggerindo, se mai minaciasse || rovina II, la mia opinione per ripararla. E questo disegno va inserto alla mia relazione del giorno di oggi.

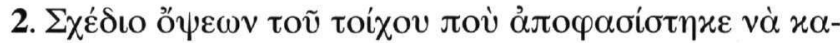

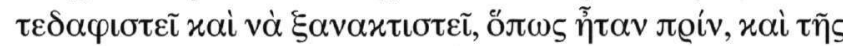

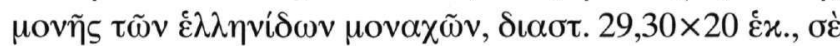

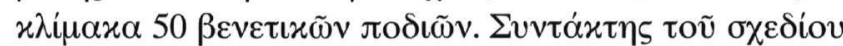

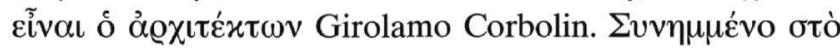

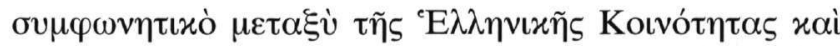

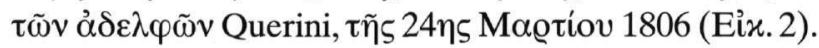

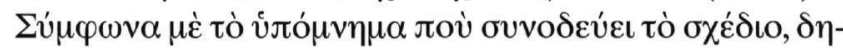

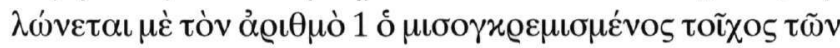

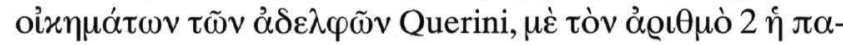

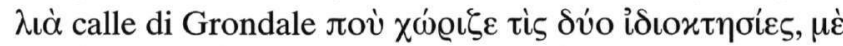

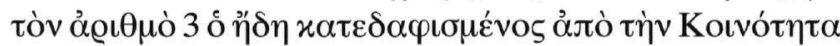

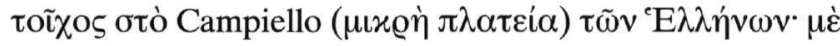

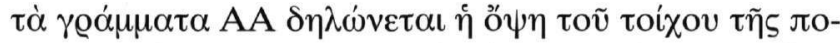

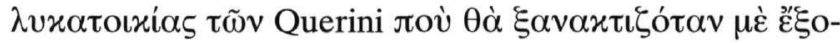

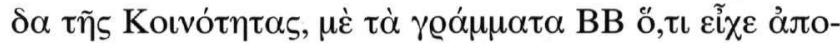

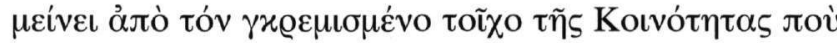

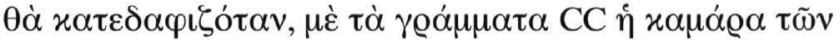

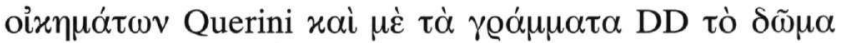

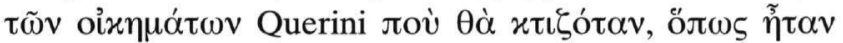

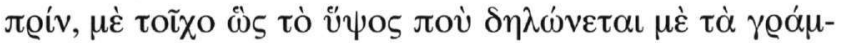
$\mu \alpha \tau \alpha \mathrm{G}$ xai $\mathrm{H}$.

a 24 marzo 1806, Venezia

Prospetto del muro maestro, riconosciuto rovinoso dall' uffiziale ingegnere del Consiglio Municipale de' Savj, il quale deve dietro all' intimazione 13 ottobre 1806, partecipata dal sunominato Consiglio alli signori Vincenzo Pietro e Fratello Querini, proprietarj dello stabile limitrofo al Campiello dei Greci, essere per intiero \{per intiero\} riattato da nuovo in tutto e per tutto simile al medesimo, vale a dire colli soli semplici sei fori marcati colle lettere $A, B, C, D, E, F$, guardanti il Campiello dei Greci anzidetto, dal capo maestro \{maestro\} muratore Valentin Sardi con quelli patti, modi e condizioni dichiariti nella carta di detto giorno che al presente resta unita; formato questo da me sottoscritto pubblico perito ed architetto per commissione dello spettabile attuale Guardiano Grande de' Greci e delli signori fratelli Querini.

In fede di che ecc.

Girolamo Corbolin, pubblico perito ed architetto, di mano propria.

\section{Chryssa Maltezou}

\section{THE WALL OF THE CAMPO DEI GRECI IN VENICE AND THE ARCHIVAL DOCUMENTATION OF ITS OWNERSHIP}

$\mathrm{T}$ vanni Veludo, in his well-known treatise on the "Ellinon orthodoxon apoikia ...", publishes an inscription, according to which the wall separating the Campo dei Greci from the adjacent apartment building was put up in 1807 and was the property of the Community. Prompted by recent claims to ownership of the wall by the tenants of the apartment building, the author carried out research in the Old Archive of the
Community, which is kept in the Hellenic Institute of Byzantine and Postbyzantine Studies in Venice, with the aim of locating archival material relating to the construction of the wall. This research brought to light documents and drawings of the period, which clearly demonstrate that the walls belongs to the inalienable property of the Community, that is the Hellenic Institute. The findings of this archival research are presented in the article. 